PHYSICAL REVIEW B 70, 219907(E) (2004)

\title{
Erratum: Theory of charge transport in diffusive normal metal/unconventional singlet superconductor contacts \\ [Phys. Rev. B 69, 144519 (2004)]
}

\author{
Y. Tanaka, ${ }^{1,2,3}$ Yu. V. Nazarov, ${ }^{3}$ A. A. Golubov, ${ }^{4}$ and S. Kashiwaya ${ }^{5}$ \\ ${ }^{1}$ Department of Applied Physics, Nagoya University, Nagoya, 464-8603, Japan \\ ${ }^{2}$ CREST Japan Science and Technology Cooperation (JST), Nagoya 464-8603, Japan \\ ${ }^{3}$ Department of Nanoscience, Faculty of Applied Sciences, Delft University of Technology, 2628 CJ Delft The Netherlands \\ ${ }^{4}$ Faculty of Science and Technology, University of Twente, 7500 AE, Enschede, The Netherlands \\ ${ }^{5}$ National Institute of Advanced Industrial Science and Technology, Tsukuba, 305-8568, Japan
}

(Received 25 September 2004; published 30 December 2004)

DOI: 10.1103/PhysRevB.70.219907

PACS number(s): 74.20.Rp, 74.50.+r, 74.70.Kn, 99.10.Cd

There are typos in the definition of several equations. We show the correct versions in the following. These are only typos in the intermediate equations, while all final results and figures are correct and are not changed.

(1) The correct expression of Eq. (11) is

$$
\check{I}=\sum_{n} \frac{2 e^{2}}{h} 2 T_{1 n}\left(1+T_{1 n}^{2}+T_{1 n}\left[\check{H}_{+}, \check{G}_{1}\right]_{+}\right)^{-1}\left[\check{H}_{+}, \check{G}_{1}\right] .
$$

(2) The correct expression of Eq. (15) is

$$
I_{b}=\frac{e^{2}}{2 h} \sum_{n} \operatorname{Tr}\left[\hat{I}_{K n} \hat{\tau}_{3}\right]
$$

with

$$
\hat{I}_{K}=\frac{2 e^{2}}{h} \sum_{n} \hat{I}_{K n}
$$

(3) Trace is missing in Eqs. (18) and (20).

(4) In Eq. (23), the definition of $\Lambda_{2}$ is given by

$$
\Lambda_{2}=\operatorname{Re}\left\{g_{+}\left(\cos \theta_{0}+\cos \theta_{0}^{*}\right)+f_{+}\left(\sin \theta_{0}+\sin \theta_{0}^{*}\right)\right\} .
$$

(5) The correct expression of Eq. (26) is given by

$$
\left.\frac{L}{R_{d}}\left[\check{G}_{N}(x) \frac{\partial \check{G}_{N}(x)}{\partial x}\right]\right|_{x=0_{-}}=-\frac{h}{2 e^{2} R_{b}}\langle\check{I}(\phi)\rangle
$$

with $\check{I}(\phi)=2 e^{2} / h \check{I}_{n 0}$.

(6) The correct definition of $R_{b}$ below Eqs. (27) and (42) is given by

$$
R_{b}=\frac{2 R_{0}}{\int_{-\pi / 2}^{\pi / 2} d \phi T(\phi) \cos \phi}
$$

with Sharvin resistance at the interface $R_{0}$.

(7) Below Eq. (32), the correct boundary condition is given by

$$
\begin{gathered}
\left.\frac{L}{R_{d}}\left(\frac{\partial f_{3 N}}{\partial x}\right) \cosh ^{2}\left[\operatorname{Im}\left(\theta_{0}\right)\right]\right|_{x=0_{-}}=-\frac{\left\langle I_{b 0}\right\rangle f_{3 N}\left(0_{-}\right)}{R_{b}}, \\
I_{b 0}=\frac{T_{n}}{2} \frac{C_{0}}{\left|\left(2-T_{n}\right)\left(1+g_{+} g_{-}+f_{+} f_{-}\right)+T_{n}\left[\cos \theta_{0}\left(g_{+}+g_{-}\right)+\sin \theta_{0}\left(f_{+}+f_{-}\right)\right]\right|^{2}} \\
C_{0}=T_{n}\left(1+\left|\cos \theta_{0}\right|^{2}+\left|\sin \theta_{0}\right|^{2}\right) \times\left[\left|g_{+}+g_{-}\right|^{2}+\left|f_{+}+f_{-}\right|^{2}+\left|1+f_{+} f_{-}+g_{+} g_{-}\right|^{2}+\left|f_{+} g_{-}-g_{+} f_{-}\right|^{2}\right]+2\left(2-T_{n}\right) \operatorname{Re}\left\{\left(1+g_{+}^{*} g_{-}^{*}+f_{+}^{*} f_{-}^{*}\right)\right. \\
\left.\times\left[\left(\cos \theta_{0}+\cos \theta_{0}^{*}\right)\left(g_{+}+g_{-}\right)+\left(\sin \theta_{0}+\sin \theta_{0}^{*}\right)\left(f_{+}+f_{-}\right)\right]\right\}+4 T_{n} \operatorname{Im}\left(\cos \theta_{0} \sin \theta_{0}^{*}\right) \operatorname{Im}\left[\left(f_{+}+f_{-}\right)\left(g_{+}^{*}+g_{-}^{*}\right)\right] .
\end{gathered}
$$

(8) In the Appendix, the definition of $v$ and $v_{n}$ is given by $v_{F}$ and $v_{F x}$, respectively. 